\title{
Orbital Angular Momentum Microlaser
}

Pei Miao, ${ }^{1 \dagger}$ Zhifeng Zhang,,${ }^{1 \dagger}$ Jingbo Sun,${ }^{1 \dagger}$ Wiktor Walasik, ${ }^{1}$ Stefano Longhi, ${ }^{2}$ Natalia M. Litchinitser, ${ }^{1 *}$ Liang Feng ${ }^{1 *}$

${ }^{1}$ Deaprtment of Electrical Engineering, The State University of New York at Buffalo, Buffalo, NY 14260, USA

${ }^{2}$ Dipartimento di Fisica, Politecnico di Milano and Istituto di Fotonica e Nanotecnologie del Consiglio Nazionale delle Ricerche, Piazza L. da Vinci 32, Milano I-20133, Italy.

†These authors contributed equally to this work.

*Emails: fengl@buffalo.edu; natashal@buffalo.edu

Structured light puts a new spin on modern optics and practical applications. Effective generation of orbital angular momentum (OAM) lasing, especially at a micro/nanoscale, while not yet demonstrated, may address the worldwide-growing demand for information capacity. By exploiting the emerging non-Hermitian photonics design at an exceptional point, we demonstrate a microring laser producing a single-mode OAM vortex lasing with ability to precisely define the topological charge of the OAM. The polarization associated with the OAM lasing can be further manipulated on-demand, creating a radially polarized vortex emission. The demonstrated OAM microlaser shows great promises towards next generation of integrated optoelectronic devices for optical communications in both quantum and classical regimes. 
Light typically consists of a stream of linearly polarized photons, traveling in a straight line and carrying a linear momentum. Since the beginning of the last century, it was recognized that beyond the linear momentum, circularly polarized light carries an angular momentum (1). However, angular momentum associated with the polarization degree of freedom, or spin angular momentum (SAM), can take only one of two values $\pm \hbar$. Only in 1990s, it was demonstrated that in addition to the SAM, a light beam can carry an orbital angular momentum (OAM) (2). Such beams possess helical phase fronts such that the Poynting vector within the beam is twisted with respect to the principle axis. This fundamental discovery of an OAM opened a new branch of optical physics, facilitating studies of physical phenomena ranging from rotary photon drag (3), angular uncertainty relationships (4), rotational frequency shifts (5) to spin-orbital coupling (6). The OAM degree of freedom has already enabled significant-technological advances, for example, edgeenhanced microscopy (7). Moreover, in contrast to the SAM that can take only two values, the OAM is unbounded. As a result, OAM beams are being considered as potential candidates for encoding information in both quantum and classical systems. The combined use of spin and orbital angular momenta is expected to enable the implementation of entirely new high-speed secure optical communication and quantum teleportation systems in a unique multidimensional space $(8,9)$, satisfying the exponentially growing demand worldwide for network capacity.

To date, most of the light sources only produce relatively simple light beams with spatially homogeneous polarization and planar wavefront. Generation of the complex OAM beams usually relies on bulk optics components, such as spiral phase plates, spatial light modulators, and computer-generated holograms (10). Although the science of the OAM light beams on the micro/nanoscale is still in its early days (11), it is likely to advance our knowledge of the interaction of light with single atoms and molecules and facilitate novel functionalities such as micromanipulation, microfluidic and lab-on-a-chip devices. Only recently the first attempts at the realization of miniaturized OAM vortex elements, including photopolymerized optical vortex converters (12), q-plates (13), and those based on metasurfaces (14-19) and silicon optical resonators (20), have been reported. Nevertheless, the existing approaches for OAM light generation are all passive and thus require an external laser source. For an ultimate miniaturized optical communication 
platform, there is a necessity of independent micro/nanoscale laser sources (21) emitting complex vector beams carrying the OAM information.

To create an OAM laser, we consider a microring cavity that is known to support whispering gallery modes (WGMs). These modes circulate inside the cavity and carry high OAM. However, because of the mirror symmetry intrinsically associated with a ring cavity, clockwise and counterclockwise eigen-WGMs are simultaneously excited and their carried OAMs consequently cancel with each other. This is evidenced by the quantized phase, taking values of either 0 or $\pi$, azimuthally distributed in the ring cavity, which results from the interference between two counter-propagating WGMs (see supplementary text and fig. S1). Hence, in order to observe the OAM of an individual WGM, it is essential to introduce a mechanism of robust selection of either clockwise or counterclockwise mode. In conventional bulk optics, such unidirectional ring lasers have been demonstrated by implementing a nonreciprocal isolator in the light path (22). The optical isolator breaks the reciprocity between counter-propagating waves in the cavity, facilitating the desired unidirectional power flow. This approach, however, is not feasible at the micro/nanoscale, as the realization of micron-sized isolators is extremely challenging by itself.

To overcome this fundamental limitation, we break the "reciprocity" by introducing in a microring cavity with complex refractive index modulations to form a so-called exceptional point (EP), as depicted in Fig. 1A. Driven by non-Hermiticity (i.e. gain and loss in optics) (23-26), EP occurs when multiple different-eigenstates coalesce into one (27-31). The microring laser resonator is designed with $500 \mathrm{~nm}$-thick InGaAsP multiple quantum wells on an InP substrate. The EP can be realized by judiciously designed modulation of the real part of the refractive index $\left(n^{\prime}\right)$ and gain/loss $\left(n^{\prime \prime}\right)$ in the cavity. Here, it is achieved by periodically introduced single-layer $\mathrm{Ge}$ and bilayer $\mathrm{Cr} / \mathrm{Ge}$ structures on top of InGaAsP along the azimuthal direction $(\theta)$, corresponding to $n^{\prime}$ and $n^{\prime \prime}$, respectively:

$$
\Delta n=\left\{\begin{array}{ccc}
i n^{\prime \prime} & \text { for } & 2 \pi p / N<\theta<2 \pi\left(p+\frac{1}{4}\right) / N, \\
n^{\prime} & \text { for } & 2 \pi\left(p+\frac{3}{8}\right) / N<\theta<2 \pi\left(p+\frac{5}{8}\right) / N
\end{array}\right.
$$

where the amplitudes of index and gain/loss modulations are set equal (i.e. $n^{\prime}=n^{\prime \prime}$ ), $N$ denotes the azimuthal number of the targeted WGM, and $p$ takes integer values from the 
set $\{0, N-1\}$. The EP modulation in Eq. (1) offers unidirectional distributed feedback (3234) to amplify only the counterclockwise WGM. As a result, the expected accidental degeneracy occurs: the clockwise WGM becomes annihilated, while the counterclockwise one unidirectionally circulates in the microring cavity carrying high OAM through the required continuous linear phase evolution along the azimuthal direction (see supplementary text and fig. S2).

The OAM associated with the unidirectional power flow is extracted upwards into free space by introducing sidewall modulations periodically arranged along the microring perimeter. The azimuthal phase dependence of the targeted unidirectional $N$ th WGM oscillation is given by $\varphi=N \theta$. The sidewall modulations are positioned such that they coherently scatter light with the phase continuously varying in azimuthal direction and uniquely defined by the locations of the scatters (see the inset of Fig. 1A). For $M$ equidistant scatters the location of the scatters is given by $\theta_{s}=2 \pi s / M$, where $s \in$ $\{0, M-1\}$, resulting in the extracted phase $\varphi_{s}=2 \pi s N / M$ that carries OAM with a continuous phase variation in the azimuthal direction. Because the physically meaningful phase is measured modulo $2 \pi$, we can thus subtract $2 \pi s$ from each of the extracted phases and derive:

$$
\varphi_{s}=2 \pi s(N-M) / M
$$

Eq. (2) shows that the extracted phase increases linearly from 0 to $2 \pi(N-M)$, therefore creating a vortex beam with topological charge $l=N-M$. Figure 1B shows the modelling result of the vortex laser emission from our OAM microring laser where $N=56$ and $M=$ 57. The phase of the electric field changes by $2 \pi$ upon one full circle around the center of the vortex. Therefore, the phase is continuous everywhere except for the center of the emission path, presenting a topological phase singularity point at the beam axis. The topological charge of the vortex emission can be viewed as the number of twist the wavefront does in one wavelength, exhibiting OAM lasing radiation of charge $l=-1$ consistent with the theoretical prediction by Eq. (2).

The OAM microring laser with the EP modulation by periodically arranged Ge and $\mathrm{Cr} / \mathrm{Ge}$ (Fig. 2) was fabricated using overlay electron beam lithography and plasma etching (see supplementary text). It is noteworthy that the unidirectional power flow oscillating in 
the cavity formed by the EP modulation intrinsically eliminates the interference pattern and thus the interference resulting spatial hole burning that causes mode competition and instability of laser output (22). Hence, in addition to the supported unidirectional power flow, the EP modulation further enables efficient and stable single-mode lasing oscillation (Fig. 3A). By increasing the optical pumping power (see supplementary text and fig. S3), the light emission showed a significant spectral narrowing from broadband photoluminescence (PL), to amplified spontaneous emission (ASE), and finally to pronounced single mode lasing with a sideband suppression ratio of approximately $40 \mathrm{~dB}$. In the transition from ASE to strong-lasing, the emission peak stabilized at the same resonant wavelength, clearly demonstrating the avoidance of mode hopping typically observed in a microring cavity. In Fig. 3B, the light-light curve of the single-mode emission demonstrated the lasing threshold corresponding to the nonlinear kink at the peak pump power density below $1 \mathrm{GW} \mathrm{m}^{-2}$. The OAM associated with the single-mode lasing, such as the vortex nature and the phase singularity, was characterized by analyzing the spatial intensity profile of lasing emission and its self-interference, as shown in Fig. 3C and Fig. $3 \mathrm{D}$, respectively. In the far field, we observed the intensity of lasing emission spatially distributed in a doughnut shape with a dark core in the center (Fig. 3C). The observed dark center is due to the topological phase singularity at the beam axis where the phase becomes discontinuous, as predicted by the simulation result in Fig. 1B. The presence of the OAM was then validated by the self-interference of two doughnut shaped beams split from the same lasing emission. In each doughnut beam, because of its associated OAM, optical phase varies more dramatically with a helical phase front close to the central singularity area, whereas the outer doughnut area is of a relatively uniform phase with a quasi-planar phase front. At the observation plane, we intentionally created a horizontal offset between two doughnut beams, such that the dark center of one beam overlapped with the bright doughnut area of the other, and vice versa. As a result, the interference patterns between the helical and quasi-planar phase fronts revealed two inverted forks (Fig. 3D), as the quasiplanar and helical phases were reversed at the centers of two doughnuts. For both of them, the single fringe split into two at the fork dislocation, evidently confirming that the radiation from our OAM laser was an optical vortex of topological charge $l=-1$. 
Moreover, the polarization properties of the demonstrated OAM microlaser can also be designed on demand. In particular, radially polarized beams, which are a subclass of cylindrical vector beams and are characterized by a nonuniform spatial distribution of their polarization vector, can be directly produced from our OAM microlaser. Radially polarized beams have enabled unique functionalities, such as high spatial resolution microscopy by their sharp focusing (35). However, conventional schemes of generating radially polarized lasing requires external optical components, such as geometric phase based diffraction elements $(14,15)$. To obtain the OAM lasing inherently associated with radial polarization, the dominant oscillating mode in our microring laser cavity is designed to be a quasi-TM mode. Compared with the azimuthally polarized component, the radial component is tightly confined at the microring perimeter and therefore sensitive to sidewall modulations, leading to a radially polarized OAM lasing (see supplementary text and fig. S4). In experiments, the polarization state of the OAM lasing was validated. After transmission through a linear polarizer, the doughnut profile observed in Fig. 3C split into two lobes aligned along the orientation of the linear polarizer (Fig. 4). The two lobes remained parallel to the polarization axis regardless of the rotation of the linear polarizer, manifesting a pure radially polarized vortex OAM lasing. Additionally, in contrast to linearly polarized OAM modes that are not compatible with optical fibers, fibers can support radially polarized OAM eigenmodes.

We have demonstrated a microring OAM laser producing an optical vortex beam with an on-demand topological charge and vector polarization states. This is enabled through the quantum physics inspired interplay between index and gain/loss modulations, which fundamentally breaks the "reciprocity" in the lasing generation dynamics and facilitates the unidirectional power oscillation. Finally, OAM vector laser beams might offer novel degrees of freedom for the next generation of optical communications in both classical and quantum regimes. 


\section{References}

1. M. Padgett, Proc. R. Soc. A 470, 20140633 (2014).

2. L. Allen, M. W. Beijersbergen, R. J. C. Spreeuw, J. P. Woerdman, Phys. Rev. A 45, 8185 (1992).

3. S. Franke-Arnold, G. Gibson, R. W. Boyd, M. J. Padgett, Science 333, 65 (2011).

4. S. Franke-Arnold et al., New J. Phys. 6, 103 (2004).

5. J. Courtial, D. Robertson, K. Dholakia, L. Allen, M. Padgett, Phys. Rev. Lett. 81, 4828 (1998).

6. Q. Guo, W. Gao, J. Chen, Y. Liu, S. Zhang, Phys. Rev. Lett. 115, 067402 (2015).

7. C. Maurer, A. Jesacher, S. Bernet, M. Ritsch-Marte, Laser Photon. Rev. 5, 81 (2011).

8. J. Wang et al., Nat. Photon. 6, 488 (2012).

9. N. Bozinovic et al., Science 340, 1545 (2013).

10. A. M. Yao, M. J. Padgett, Adv. Opt. Photon. 3, 161 (2011).

11. N. M. Litchinitser, Science 337, 1054 (2012).

12. G. Knöner et al., Opt. Express 15, 5521 (2007).

13. E. Karimi, B. Piccirillo, E. Nagali, L. Marrucci, E. Santamato, Appl. Phys. Lett. 94, $231124(2009)$.

14. Z. Bomzon, G. Biener, V. Kleiner, E. Hasman, Opt. Lett. 27, 1141 (2002).

15. Z. Bomzon, V. Kleiner, E. Hasman, Appl. Phys. Lett. 79, 1587 (2001).

16. N. Yu et al., Science 334, 333 (2011).

17. D. Lin, P. Fan, E. Hasman, M. L. Brongersma, Science 345, 298 (2014).

18. K. E. Chong et al., Nano Lett. 15, 5369 (2015).

19. M. I. Shalaev et al., Nano Lett. 15, 6261 (2015).

20. X. Cai et al., Science 338, 363 (2012).

21. M. A. Noginov et al., Nature 460, 1110 (2009).

22. A. E. Siegman, Lasers, (University Science Books, 1986).

23. K. G.Makris, R. El-Ganainy, D. N. Christodoulides, Z. H. Musslimani, Phys. Rev. Lett. 100, 103904 (2008).

24. C. E. Rüter et al., Nat. Phys. 6, 192 (2010).

25. L. Feng, Z. J. Wong, R. M. Ma, Y. Wang, X. Zhang, Science 346, 972 (2014). 
26. H. Hodaei, M. A. Miri, M. Heinrich, D. N. Christodoulides, M. Khajavikhan, Science 346, 975 (2014).

27. J. Wiersig, S. W. Kim, M. Hentschel, Phys. Rev. A 78, 053809 (2008).

28. B. Peng et al., Nat. Phys. 10, 394 (2014).

29. H. Schomerus, Phys. Rev. Lett. 104, 233601 (2010).

30. M. Brandstetter et al., Nat. Commun. 5, 4034 (2014).

31. L. Ge, A. D. Stone, Phys. Rev. X 4, 031011 (2014).

32. Z. Lin et al., Phys. Rev. Lett. 106, 213901 (2011).

33. A. Regensburger et al., Nature 488, 167 (2012).

34. L. Feng et al., Nat. Mater. 12, 108 (2013).

35. R. Dorn, S. Quabis, G. Leuchs, Phys. Rev. Lett. 91, 233901 (2003). 


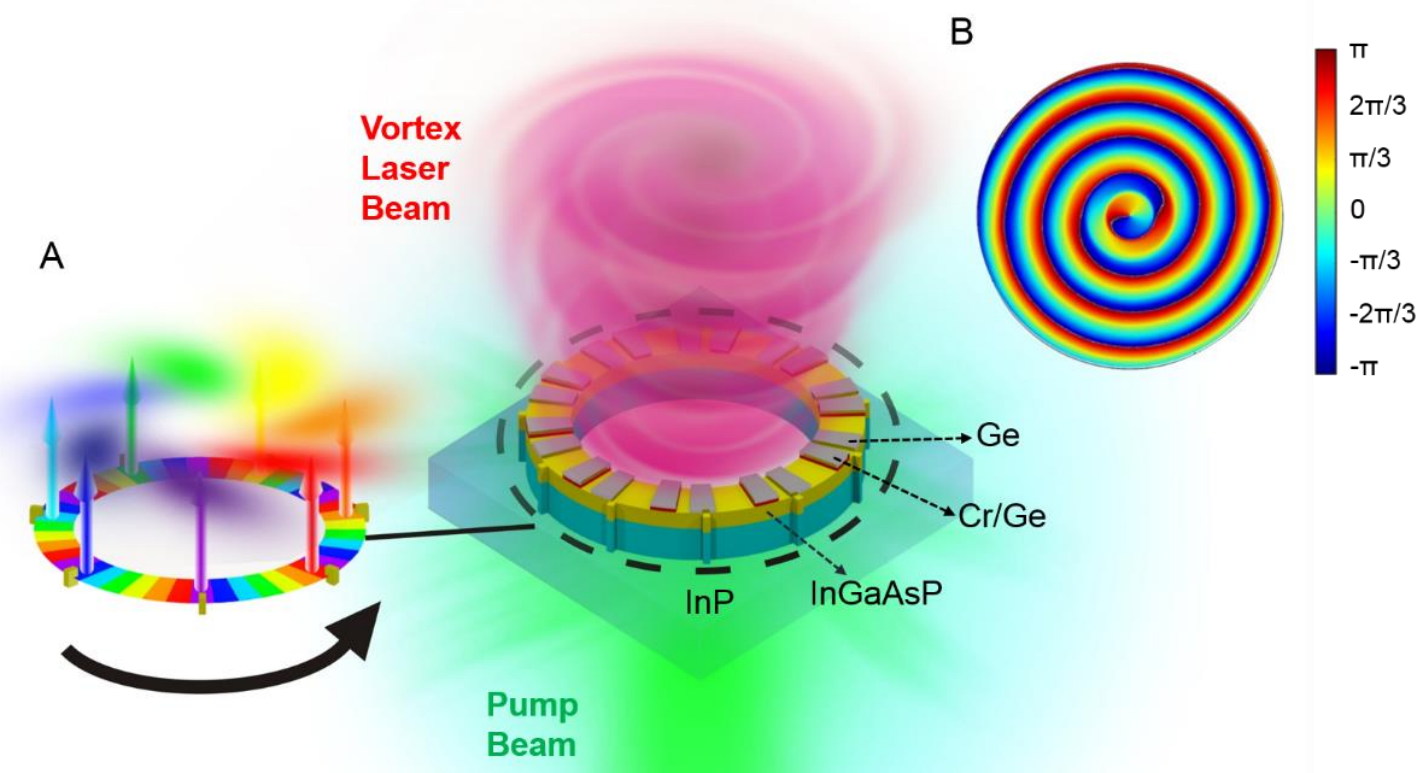

Figure 1. Design of OAM microring laser. (A) Schematic of the OAM microring laser on an InP substrate: the diameter of the microring resonator is $9 \mu \mathrm{m}$, the width is $1.1 \mu \mathrm{m}$ and the height is $1.5 \mu \mathrm{m}$ (500 $\mathrm{nm}$ of InGaAsP and $1 \mu \mathrm{m}$ of InP). $13 \mathrm{~nm}$ Ge single-layer and $5 \mathrm{~nm} \mathrm{Cr} / 11 \mathrm{~nm}$ Ge bilayer structures are periodically arranged in the azimuthal direction on top of the InGaAsP/InP microring resonator, mimicking real index and gain/loss parts, respectively, of an EP modulation at $n^{\prime}=n^{\prime \prime}=0.1$ and thus supporting a unidirectional power flow in the cavity. Here, the designed azimuthal order is $N=56$ at the resonant WGM wavelength of $1472 \mathrm{~nm}$. Equidistant sidewall scatters with a total number of $M=$ 57 are introduced to couple the lasing emission upwards with the precisely controlled extracted phases, creating an OAM vortex emission with a helical wavefront. Its topological charge is defined by $l=N-M=-1$. (B) Simulated phase distribution of the emitted light. A spiral phase distribution characteristic for an OAM charge one vortex is clearly demonstrated. 

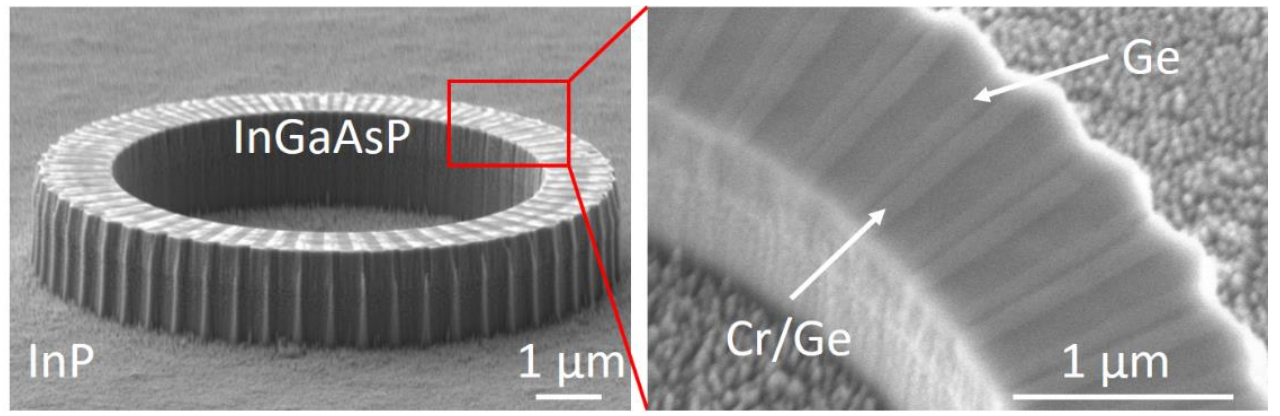

Figure 2. Scanning electron microscope images of OAM microring laser. The EPmodulated microring laser was fabricated on the InGaAsP/InP platform with sidewall modulations to extract the OAM lasing. Alternating $\mathrm{Cr} / \mathrm{Ge}$ bilayer and Ge single-layer structures were periodically implemented in the azimuthal direction on top of the microring resonator, presenting respectively the index and gain/loss modulations required for unidirectional power flow circulating in the microring. 

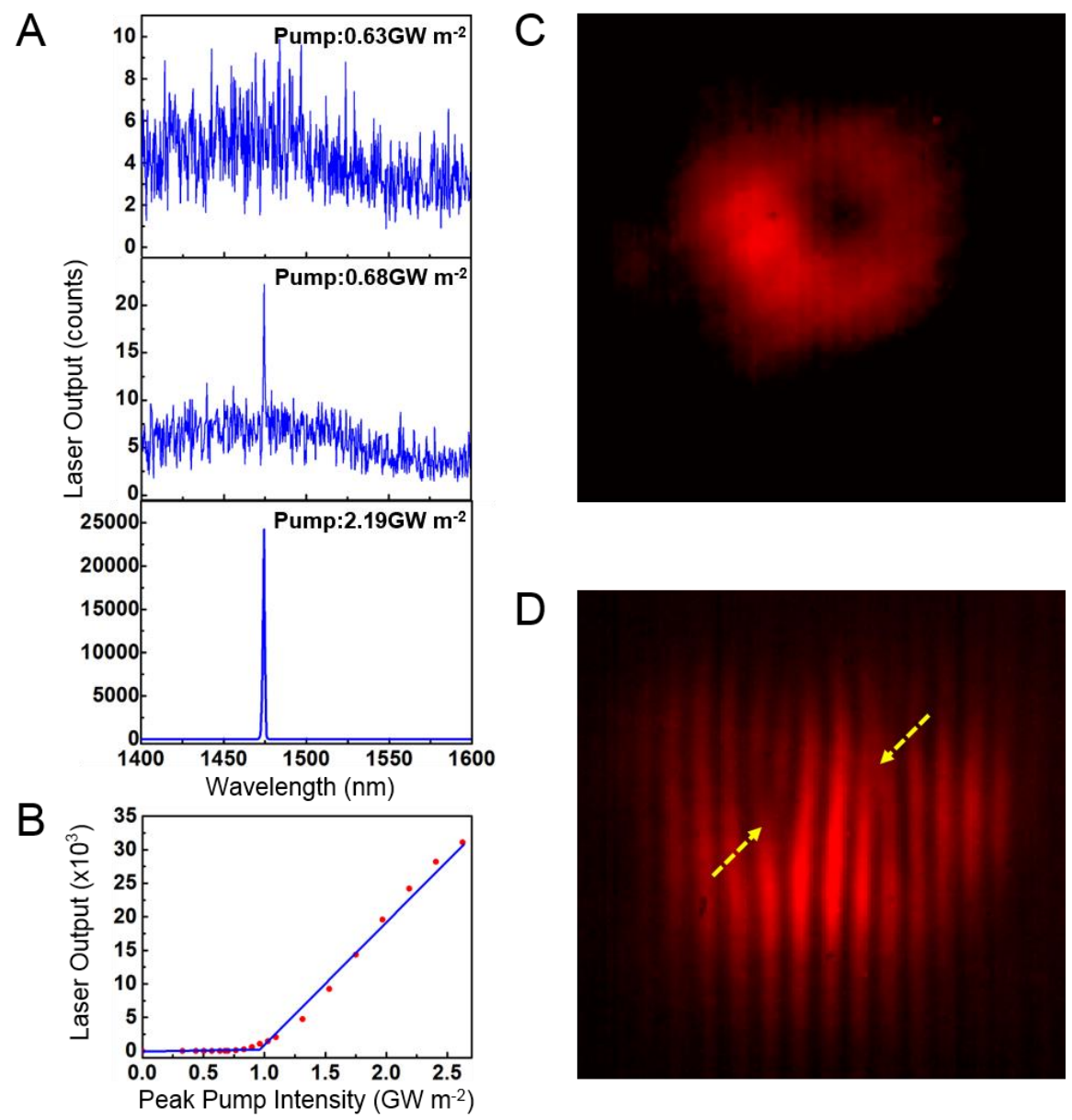

Figure 3. Experimental characterization of OAM lasing. (A) Evolution of the light emission spectrum from PL, to ASE, and to single-mode lasing at the resonant wavelength of $1474 \mathrm{~nm}$, as the peak power density of pump light was increased from $0.63 \mathrm{GW} \mathrm{m}^{-2}$, $0.68 \mathrm{GW} \mathrm{m}^{-2}$, to $2.19 \mathrm{GW} \mathrm{m}^{-2}$, respectively. (B) The power relationship between the lasing emission and the pump light, where a clear onset of the single-mode lasing appears at a threshold of approximately $1 \mathrm{GW} \mathrm{m}^{-2}$. (C) Far field intensity distribution of the laser emission exhibiting a doughnut shaped profile, where the central dark core is due to the phase singularity at the center of the OAM vortex radiation. (D) Off-center selfinterference of the OAM lasing radiation, showing two inverted forks (marked with arrows) located at two phase singularities. Originating from the superposition of central helical and outer quasi-planar phases intrinsically associated with OAM, the double-fork pattern confirms the OAM vortex nature of the laser radiation. Each fork splits into two branches, validating a topological charge of $l=-1$. 

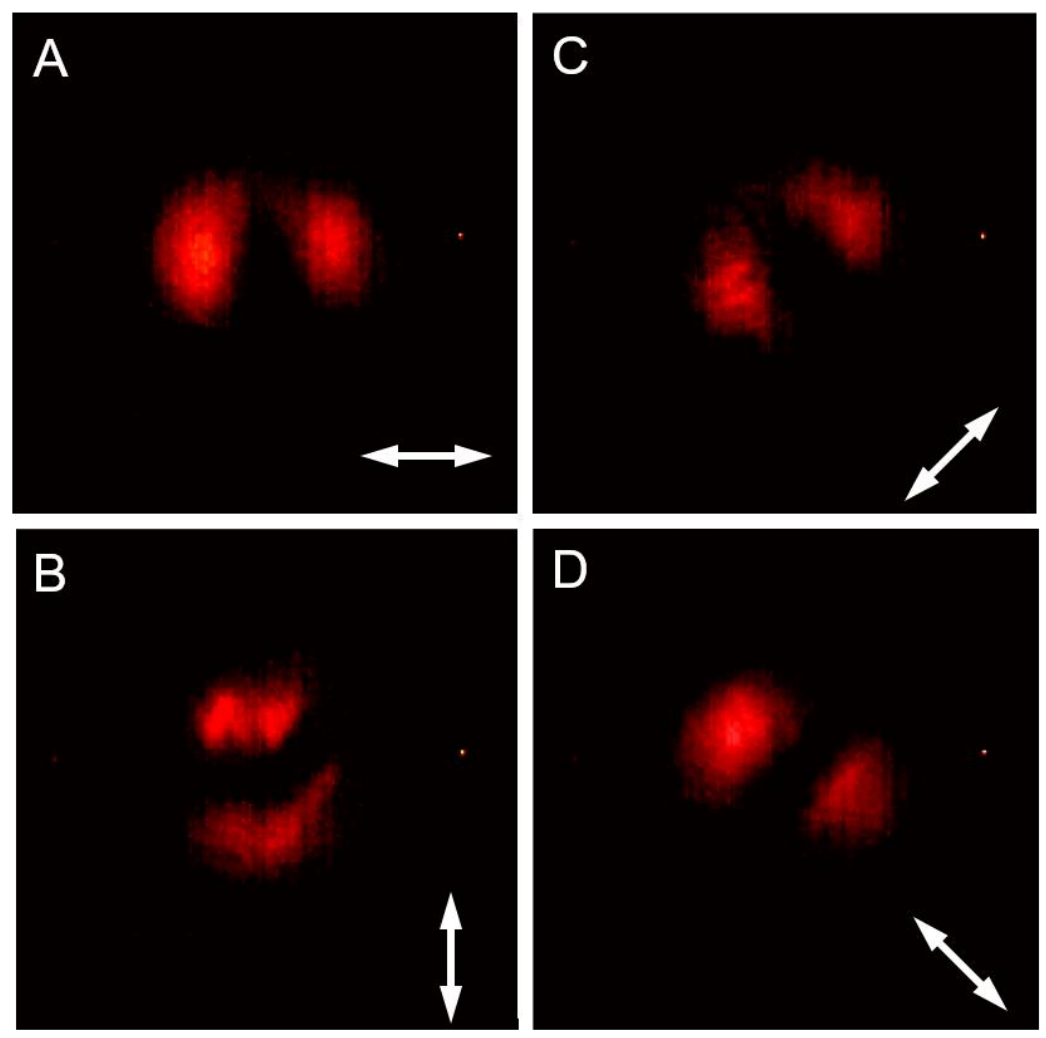

Figure 4. Polarization state of OAM lasing. Measured intensity distributions of the OAM lasing radiation passing through a linear polarizer with different polarization orientations indicated by arrows: (A) $0^{\circ}$, (B) $90^{\circ}$, (c) $45^{\circ}$, and (D) $-45^{\circ}$. A two-lobe structure aligned with the polarizer orientation was observed. This two-lobe structure rotated with the rotation of the polarizer in the same fashion, confirming a pure radially polarized OAM lasing. 